Classification

Physics Abstracts

$64.70 \mathrm{Md}-6110 \mathrm{Lx}-82.20 \mathrm{Mj}$

\title{
Rates of Phase Transformations between Mesophases Formed by a Non-Ionic Surfactant in Water: a Time-Resolved X-Ray Diffraction Study
}

\author{
Marianne Clerc $\left({ }^{1}\right)$, Peter Laggner $\left({ }^{2}\right)$, Anne-Marie Levelut $\left({ }^{1}\right)$ and Gert Rapp $\left({ }^{3}\right)$ \\ ${ }^{1}$ ) Laboratorre de Physique des Solides, Bâtiment 510, Unıversité Paris-Sud, 91405 Orsay, \\ France \\ $\left({ }^{2}\right)$ Institute of Biophysics and X-ray Structure Research, Austrian Academy of Sciences, Steyr- \\ ergasse 17, $8010 \mathrm{Graz}$, Austria \\ ( $\left.{ }^{3}\right)$ EMBL Outstation at DESY, Notkestraße 85, 22603 Hamburg, Germany
}

(Received 1 February 1995, recelved in final form 5 April 1995, accepted 10 Aprıl 1995)

Résumé. - On étudie les transitions de phase hexagonale/cubique et cubique/lamellaire du système binarre $\mathrm{C}_{12} \mathrm{EO}_{6} / \mathrm{eau}$, par une technique de saut en température. Un laser IR pulsé produit un saut en température, et l'évolution temporelle d'un échantillon est suive par diffraction des rayons $\mathrm{X}$ résolue en temps toutes les $50 \mathrm{~ms}$. La cinétique de ces deux transitions de phase a un temps caractéristique pouvant varier de l'échelle de temps d'une seconde à moins de $100 \mathrm{~ms}$, selon la valeur de la température avant le saut de température et de la puissance délivrée par le laser IR. L'évolution temporelle de l'intensité diffractée est reliée à celle des fractions de volume des deux phases durant une transition. Les résultats sont interprétés dans le cadre d'un processus de nucléation/croissance.

\begin{abstract}
We studied the hexagonal/cubic and cubic/lamellar phase transitions of the $\mathrm{C}_{12} \mathrm{EO}_{6} /$ water binary system initiated by a mill temporal evolution of the structural changes were recorded using synchrotron X-ray diffraction with $50 \mathrm{~ms}$ time-resolution The time constants of both phase transitions vary from seconds to less than $100 \mathrm{~ms}$, according to the initial temperature prior to the $T$-jump and to the power of the IR laser. The intensity of the X-ray reflections is related to the volume fractions of both phases during a phase transformation. An interpretation of the results in the framework of a nucleation and growth mechanism is given.
\end{abstract}

\section{Introduction}

The binary mixture of the non-ionic surfactant $\mathrm{C}_{12} \mathrm{EO}_{6}$ with water $[1,2]$ is a well-known generic example of lyotropic liquid-crystal, and has been extensively studied. Around $65 \%$ by weight of the $\mathrm{C}_{12} \mathrm{EO}_{6}$ surfactant in water, the phase diagram [2] (Fig. 1) exhibits a sequence of three mesophases: a $2 \mathrm{D}$ direct hexagonal phase at low temperatures, a $3 \mathrm{D}$ bicontinuous cubic phase 


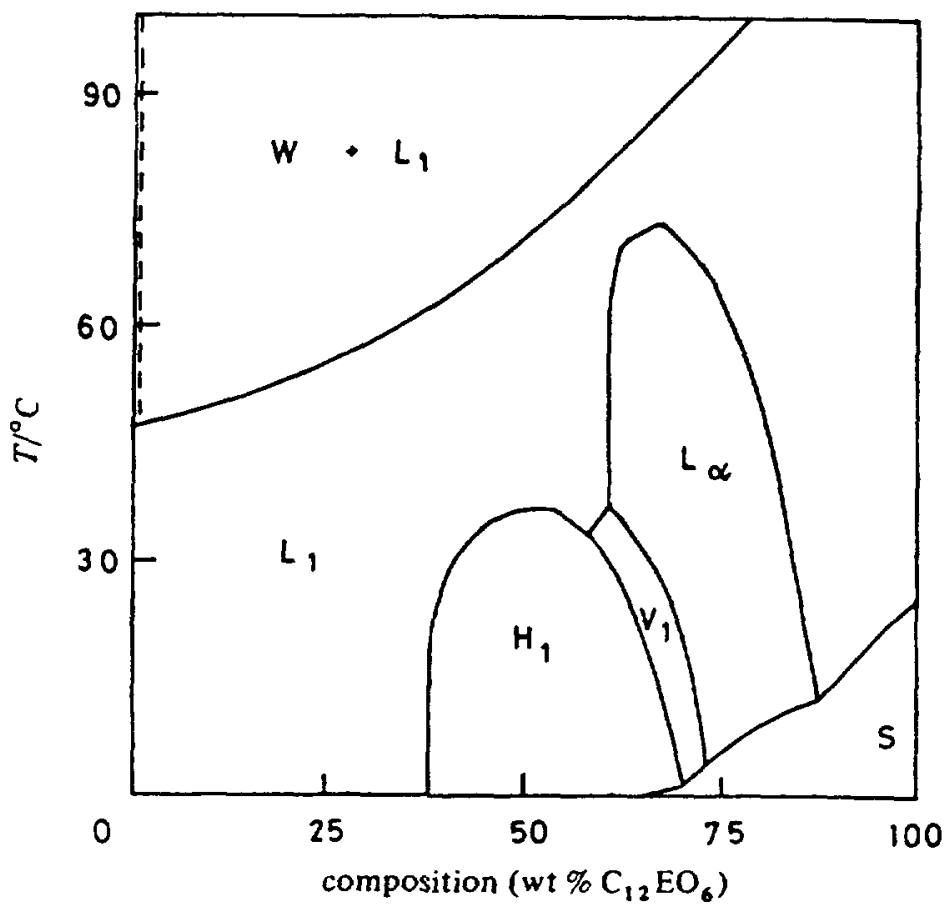

Fig. 1. - The binary phase diagram of the non-ionic surfactant $\mathrm{C}_{12} \mathrm{EO}_{6}$ in water, reprinted from [2].

(space group Ia3d) and a 1D lamellar phase at high temperatures. The structure of the cubic phase consists of an infinite 3D-periodic water film, dividing the space into two equivalent subspaces containing the surfactant molecules [3,4].

In this system large single domains of the cubic phase can grow quite easily [5]. A structural relationship, called an epitaxial relation, between electron dense planes in the three mesophases was found $[3,4]$. The $d_{100}$ spacing in the hexagonal phase, the $d_{211}$ in the cubic and the $d_{100}$ in the lamellar are equal [6]. The hexagonal/cubic and cubic/lamellar transitions are first-order ones, as shown by the existence of biphasic regions and by a small density discontinuity, $\Delta \rho$ (estimation from [1] gives $\Delta \rho \leq 10^{-4} \mathrm{~g} \mathrm{~cm}^{-3}$ ).

Using the epitaxial relations, some geometrical constructions have shown that both phase transitions need to involve only local rearrangements of the structures (connections of cylinders in the hexagonal phase or junctions of bilayers in the lamellar) [4]. The aim of this study is to test whether the epitaxial relations govern the growth mechanism of the mesophases under non-equilibrium conditions induced by an infrared laser temperature jump which increases the temperature of the sample both rapidly (within $2 \mathrm{~ms}$ ) and homogeneously. A $T$-jump study has been performed on the $\mathrm{C}_{12} \mathrm{EO}_{6}$ /water system [7] using Joule heating and observing the optical birefringence of the sample (hexagonal and lamellar phases are birefringent but the cubic phase is optically isotropic). The characteristic response times for the two phase transitions have been found to be in the range of a few seconds. In our study we measured the structural changes following a $T$-jump using synchrotron X-ray diffraction. This technque proved to be a powerful tool to study phase transitions in aqueous dispersions of phospholipids $[8,9]$ showing structural intermediates under non-equilibrium conditions not seen in transitions close to equilibrium, i.e., under slow scan conditions. 
In the following sections we shall describe our experimental approach. The response of the sample to different initial temperatures prior to the $T$-jump and to different laser energies will be analysed. Finally, we give an interpretation of the results in the framework of a nucleation and growth mechanism.

\section{Materials and Methods}

2.1. Sample Preparation. - $\mathrm{C}_{12} \mathrm{EO}_{6}$ was purchased from Nikko Chemical [10] and used without further purification. Distilled water was mixed with the surfactant and equilibrated for a few days at about $40{ }^{\circ} \mathrm{C}$. Concentrations of $62.1 \%$ and $65 \%(\mathrm{w} / \mathrm{w})$ of $\mathrm{C}_{12} \mathrm{EO}_{6}$ in $\mathrm{H}_{2} \mathrm{O}$ were used. For the $\mathrm{X}$-ray experiments the samples were placed in Lindeman cylindrical glass capillaries $(\oslash 1 \mathrm{~mm}$ and $1.5 \mathrm{~mm}$ ).

As pointed out in the introduction, large single domains of the cubic phase spontaneously appear in these samples, which results in asymmetric diffraction patterns with respect to peak intensities due to the anisotropic distribution of the orientation of the domains in the sample. To collect diffraction data using a 1D detector, i.e., to have a "powder" diffraction configuration the capillaries were rotated around their axis during the experiment using a small $\mathrm{DC}$ motor designed for this purpose. Its speed was fixed to 1320 revs per min, equivalent to a complete rotation in $45 \mathrm{~ms}$ (at higher speeds the capillaries break easily). The recorded patterns are symmetric in this configuration as checked by collecting $2 \mathrm{D}$ patterns using a Fuji image plate system. However, the absolute intensities of the diffraction peaks are not completely reproducible from sample to sample as a residual effect of the anisotropic domain orientation.

2.2. LASER TemperatURE-JUMP. - For rapid heating of aqueous samples a variety of techniques are available $A$ discussion of these and selection criteria for the individual systems are given in $[11,12]$ together with a description of the set-up used for these experiments. In brief, we used an erbium: glass laser operating at $\lambda=1.54 \mu \mathrm{m}$ where the linear absorption coefficient of water is $6.5 \mathrm{~cm}^{-1}[13]$. The sample is heated within $2 \mathrm{~ms}$ by as much as $12^{\circ} \mathrm{C}$ depending on the laser energy, which was adjustable within a range of about $0.5 \mathrm{~J}-1 \mathrm{~J}$. Preliminary IR absorbance measurements [14] of our samples indicate, as expected, that the major contribution to the absorbance at this wave-length arises from water rather than from $\mathrm{C}_{12} \mathrm{EO}_{6}$.

2.3. Data Collection and Analysis. - The small-angle $\mathrm{X}$-ray diffraction experiments were performed at beam line X13 of the EMBL outstation at DESY. One-dimensional diffraction patterns were recorded using a sealed linear detector with delay line readout [15] as part of the standard data acquisition system [16], which was controlled by a PC. Excess radiation was avoided using a small solenoid driven shutter close to the sample. Additional information, like temperature, ring current and X-ray flux measured with an ionisation chamber in front of the sample, was stored in the local memory. The reciprocal spacings $s=1 / d=(2 / \lambda) \sin \theta$ ( $d$ : lattice spacing, $2 \theta$ : scattering angle, $\lambda$ : wavelength of radiation) were calibrated by the diffraction pattern of dry rat-tail collagen with a long spacing of $65 \mathrm{~nm}$.

The sample was in a temperature controlled holder with mylar windows. The laser beam was directed onto the sample approximately collinearly with the X-ray beam. It was reflected back to the sample by an X-ray transparent aluminium coated kapton foil to (i) increase the jump amplitude and (ii) reduce thermal gradients in the sample. Diffraction patterns were recorded on a $1 \mathrm{D}$ gas detector every $50 \mathrm{~ms}$ prior to and after the laser flash. Usually the beam intensity was strong enough to obtain a sufficient signal to noise ratio even in one $T$-jump 


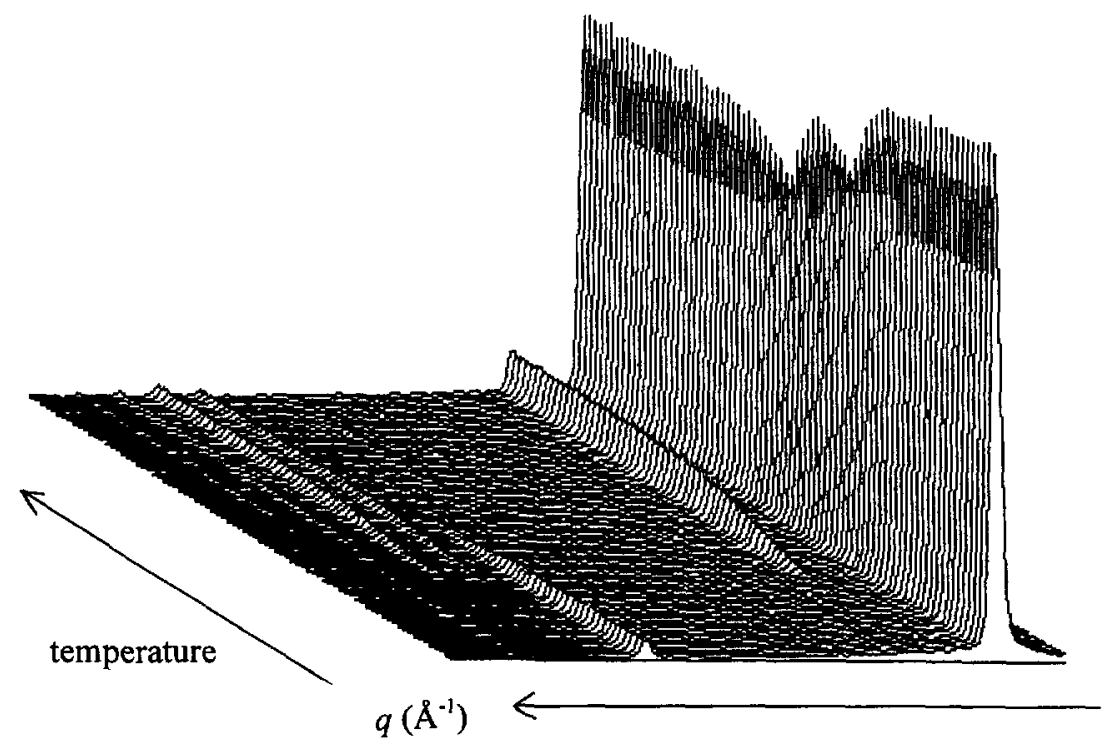

Fig. 2. - Hexagonal/cubic phase transition close to equilibrium. A slow temperature scan $\left(07^{\circ} \mathrm{C}\right.$ per min) of the sample B between $T=18.5^{\circ} \mathrm{C}$ and $T=30^{\circ} \mathrm{C}$ is performed. A 3D-plot of the X-ray scattering collected on the 1D gas detector for different temperatures is shown. Only one half of the diffraction pattern is shown. Characteristic diffraction peaks of both phases are successively observed. At low temperature, the first and the $\sqrt{3}$ hexagonal reflections are present. The two-phase region is located between $T=23.4^{\circ} \mathrm{C}$ and $T=28{ }^{\circ} \mathrm{C}$ : in that temperature range, the $\sqrt{3}$ hexagonal reflection and the $\sqrt{8}$ cubic reflection are both observed. At higher temperatures, several reflections of a pure cubic phase $(\sqrt{6}, \sqrt{8} .$.$) are observed.$

experiment. In some cases several $T$-jump records of the same experiment were averaged after it was verified that they are similar.

Data were normalized for the ionisation chamber and analysed using the interactive data evaluation program OTOKO [17] The fitting of the data by exponential functions was performed using the program ACE/gr (xmgr) [18].

\section{Results}

3.1. Transitions Close to Equilibrium. - First of all, we perform static measurements on each sample before doing any $T$-jumps. We increase the temperature at a rate of about $1^{\circ} \mathrm{C} / \mathrm{min}$ and record a series of diffraction patterns (Fig. 2). To avoid any metastability these scans were always made by heating the sample. In the case of the cubic/lamellar transition, this is particularly important, because a metastable hexagonal phase can be induced by a slow cooling of the lamellar phase, before the appearance of the cubic phase, as observed for the first time in [3]. After a $T$-jump from the cubic phase to the lamellar phase, the cubic phase always reappears during the cooling of the sample, as expected from the phase diagram: the rate of cooling is in that case not slow enough to induce the metastable hexagonal phase.

3.2. Time Response of a Sample to a $T$-Jump. - A typical result is shown in Figure 3. On the first record immediately after the $T$-jump, a change in the diffraction pattern is observable (there is no dead-time in the resolution of the experiment). The phase transition from 

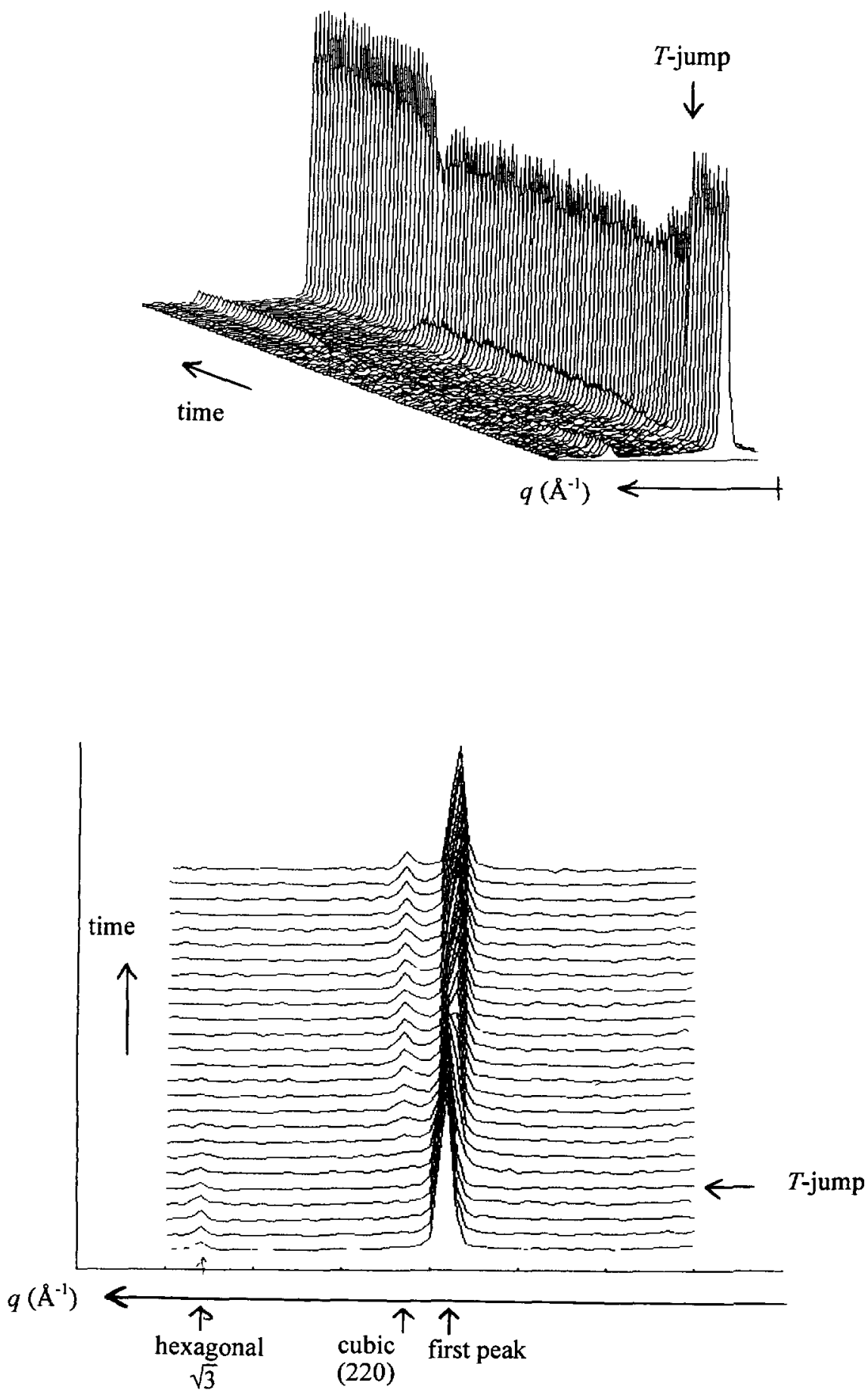

Fig. 3. - Hexagonal/cubic T-jump: 3D-plot of the X-ray scattering collected on the $1 \mathrm{D}$ gas detector versus time. Only one half of the diffraction pattern is shown After the $T$-jump, a hexagonal to cubic phase transformation is induced. The $\sqrt{3}$ hexagonal reflection disappears and the $\sqrt{8}$ cubic reflection appears at the same rate (see Section 2). No intermediate structure is observed. The small discrepancy between the locations of the first peak of the two phases is clearly visible: the epitaxial relation is not exactly verified. 
Table I. - Two-phase regions. $T_{\text {trans }}$ is the lowest temperature at which the coexistence of the two phases is observed. $T_{\text {up }}$ is the lowest temperature at which the low-temperature phase is no longer observed. $\Delta T=T_{\text {up }}-T_{\text {trans }}$ is the extension of the two phase region. Notzce that the real concentrations of the samples (capillaries) are slightly different from the bulk solutions: small amounts of water can evaporate from a capullary before the measurements. The values of the $T_{\text {trans }}$ cubuc/lamellar show that the concentration of the sample $C$ is between the concentrations of $A$ and $B: T_{\text {trans }}$ cubic/lamellar decreases with the surfactant concentration.

\begin{tabular}{|c|c|c|c|c|c|c|c|}
\hline \multirow{3}{*}{\begin{tabular}{|c|} 
sample \\
A \\
\end{tabular}} & \multirow{3}{*}{$\begin{array}{c}\text { concentration } \\
\text { (\% wetght) } \\
621\end{array}$} & \multirow[b]{2}{*}{$T_{\text {trans }}$} & \multicolumn{2}{|c|}{ hexagonal/cubic } & \multicolumn{3}{|c|}{ cubic/lamellar } \\
\hline & & & $T_{\text {UD }}$ & $\Delta \mathrm{T}$ & $T_{\text {trans }}$ & $T_{\text {up }}$ & $\Delta \mathrm{T}$ \\
\hline & & & & & 446 & 449 & 03 \\
\hline B & 65 & $\begin{array}{r}234 \\
226 \\
\end{array}$ & $\begin{array}{r}280 \\
286 \\
\end{array}$ & $\begin{array}{l}46 \\
60 \\
\end{array}$ & 359 & 379 & 20 \\
\hline C & 621 & $235 \pm 05$ & & & 364 & 379 & 15 \\
\hline
\end{tabular}

a hexagonal to a cubic phase is clearly observed: the intensity of the $\sqrt{3}$ hexagonal diffraction peak decreases with time and, at the same rate, the $\sqrt{8}$ ((220) reflection) of the cubic phase increases. The "first" peak intensity varies with time at the same rate as the others. Notice that this first peak stems, as expected from the epitaxial relation, from the superposition of the first-order peaks of the two phases: the (100) hexagonal reflection and the (211) cubic one. A detailed study indicates in fact a small discrepancy between the positions of these two first-order peaks, revealing a small "violation" of the epitaxial relation, as explained below (see "epitaxial relation"). No continuous displacement of a peak position is observed. After about $2 \mathrm{~s}$, a pure cubic phase diffraction pattern is obtanned No intermediate structure is involved in this transition. After about $30 \mathrm{~s}$ the hexagonal phase reappears, revealing the cooling of the sample back to the initial temperature prior to the laser $T$-jump: the energy of the IR laser pulse has dissipated into the sample environment by thermal diffusion. Similar results are obtained in the case of the cubic to lamellar transition.

In Section 3, we show that the characteristic time of the phase transformation varies from about $1.5 \mathrm{~s}$ to less than $100 \mathrm{~ms}$, depending on the values of the initial temperature and on the IR laser power. Before that, we devote the last section to the measurements of the lattice spacings of the different phases, in order to test the epitaxial relation.

3.3. Two-Phase Regions. - - Two phase regions are recorded during the temperature scans close to equilibrium when reflections of both phases coexist (Fig. 2). $T_{\text {trans }}$ is defined as the lowest temperature at which coexistence is observed. $\Delta T$ is the width of a two phase region. Data on the two phase regions of the samples are given in Table I. Notice that these regions are not drawn on the phase diagram of [2] but our present results show that their widths are not negligible In the first published phase diagram [1], the two phase regions were reported, 
but the cubic phase is considered there as some part of the isotropic phase, so one has to be careful in using this phase diagram.

Hexagonal/cubic two phase regions are broader (around $5{ }^{\circ} \mathrm{C}$ ) than the cubic/lamellar ones (less than $2^{\circ} \mathrm{C}$ ) in the concentration range of our study.

3.4. Epitaxial Relation. - The lattice spacings $d$ of the phases are measured first during the static experiments (Fig. 4). We define for each phase $d$ as follows: $d=d_{100}$ in the hexagonal phase, $d=d_{211}$ in the cubic one and $d=d_{100}$ in the lamellar one. $d$ is shown in Figure 4 instead of the parameters $a_{\mathrm{i}}$ of the different phases $\left(a_{\mathrm{hex}}=\frac{2 d}{\sqrt{3}}, a_{\mathrm{cub}}=\sqrt{6} d\right.$, $a_{\text {lam }}=d$ ) in order to test the epitaxial relation.

$d$ increases slowly with temperature along the sequence of the three phases. No discontinuity is observed on crossing the cubic/lamellar two phase region $(d=47.5 \pm 0.2 \AA)$. In the case of the hexagonal/cubic transition, a clear discontinuity in $d$ is measured: $d=46.4 \pm 0.2 \AA$ just below $T_{\text {trans }}$ and $d=47.4 \pm 0.2 \AA$ just above $T_{\text {trans }}+\Delta T$.

It is of importance to compare the spacings $d$ of slow scan experiments with rapid $T$-jumps (F1g. 4). As expected, $d$ is larger after the $T$-jump than before. The changes in the $d$ values are consistent with the static measurements. In comparison with the static case, we conclude that a $T$-jump can induce a high temperature phase located above the two phase region. By increasing the IR laser power, $d$ increases slightly, which shows that the induced phase corresponds to a higher temperature.

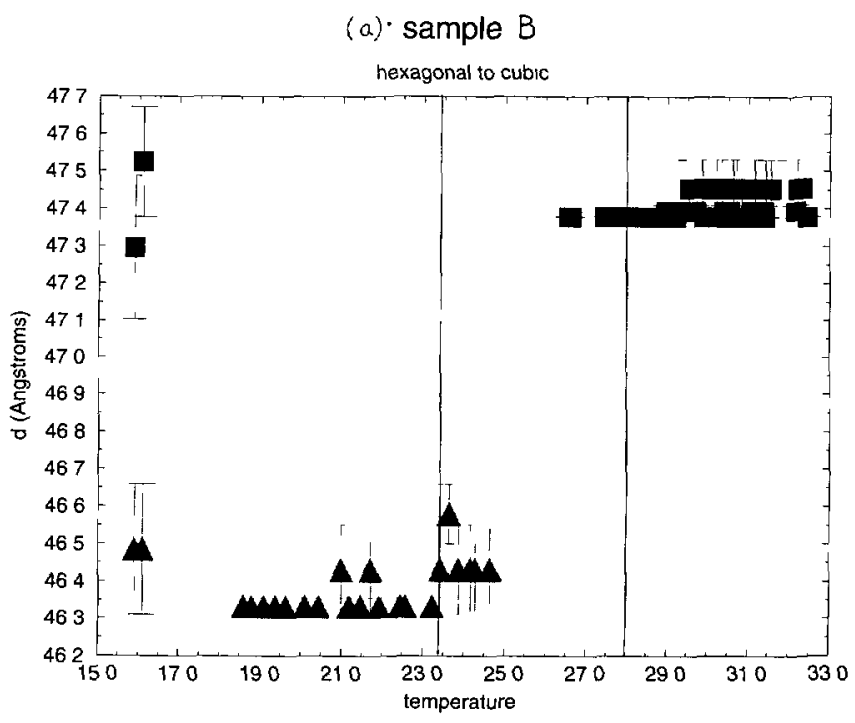

Fig. 4. - $d$ spacings versus temperature: comparison of static measurements and $T$-jump experiments. $\Delta d$ is the difference between the value of $d$ just after and just before a transition.

a) Sample B (see Tab. I): hexagonal/cubic transition. (•) static measurements: $T_{\text {trans }}=23.4{ }^{\circ} \mathrm{C}$, $T_{\text {up }}=28.0^{\circ} \mathrm{C}: \Delta d=1.0 \AA ;(\Lambda)$ pure hexagonal phase: $d=46.4 \pm 0.2 \AA ;(\mathbf{\square})$ pure cubic phase:

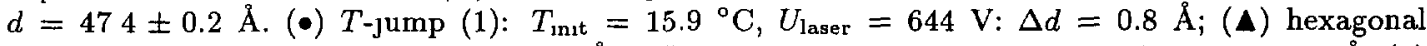
phase before the $T$-jump: $d=465 \pm 02 \AA ;(\mathbb{C})$ cubic phase after the $T$-jump: $d=47.3 \pm 0.2 \AA$. $(\bullet)$ $T$-jump (2): $T_{\mathrm{tm} t}=16 .{ }^{\circ} \mathrm{C}, U_{\text {laser }}=720 \mathrm{~V}: \Delta d=10 \AA ;(\Lambda)$ hexagonal phase before the $T$-jump: $d=46.5 \pm 0.2 \AA$. (D) cubic phase after the $T$-jump: $d=47.5 \pm 0.2 \AA$. 


\section{(b): sample C}

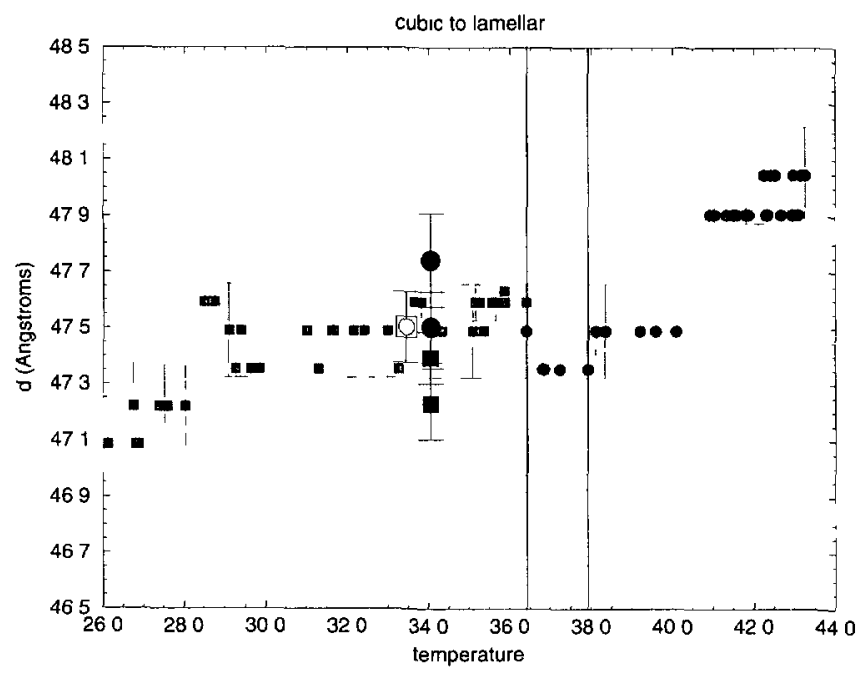

Fig. 4. - b) Sample C (see Tab I): cubıc/lamellar transition. (•) static measurements: $T_{\text {trans }}=$ $36.4^{\circ} \mathrm{C}, T_{\text {up }}=37.9^{\circ} \mathrm{C}: \Delta d=0.0 \AA$, (⿴) pure cubic phase: $d=47.5 \pm 0.2 \AA ;(\bullet)$ pure lamellar phase: $d=47.5 \pm 0.2 \AA(\bullet) T$-jump $(1) \cdot T_{\mathrm{nnt}}=33.5^{\circ} \mathrm{C}, U_{\text {laser }}=650 \mathrm{~V} \cdot \Delta d=0.0 \AA ;(\square)$ cubic phase before the $T$-jump: $d=47.5 \pm 0.2 \AA,(\bigcirc)$ lamellar phase after the $T$-jump: $d=47.5 \pm 0.2 \AA$. (•) $T$-jump (2): $T_{\text {nit }}=34.0^{\circ} \mathrm{C}, U_{\text {laser }}=820 \mathrm{~V}: \Delta d=0.4 \AA$; $($ cubic phase before the $T$-jump: $d=47.3 \pm .02 \AA ;$ (๑) lamellar phase after the $T$-jump: $d=47.7 \pm 0.2 \AA$.

With the resolution of the beam-line the epitaxial relation can be tested. For the first time, a "violation" of this relation in the case of the hexagonal/cubic transition of $2 \%$ is measured. An upper limit of $0.5 \%$ (the resolution of our measurement) in the case of the cubic/lamellar transition can be given. In the case of very "efficient" $T$-jumps, this violation is even stronger because of the increase of $d$ with temperature. However, these effects are small $(<3 \%)$ and it is still appropriate to talk of an epitaxial relation.

One has to bear in mind that, during the phase transition, a small discontinuity in density $\Delta \rho$ exists, as expected for first-order transitions. Presumably this density discontinuity is the origin of the different spacings $\Delta d$ observed. However, this assumption would require that the $d$-spacings defined for different phases correspond to phases of the same density, which is not obvious a priori.

3.5. Influence of the Initial Temperature and of the IR Laser Power on the KINETICS. - To quantify the rate of a phase transformation, we selected the temporal evolution of the intensity of the first peak. Notice that this peak contains the major part of the diffracted intensity. The other peaks have much smaller intensities with poorer statistics. We integrate the intensity of the first peak and call it $I(t)$. One has to remember that $I(t)$ contains the contributions of the two phases. Several $I(t)$ curves are shown in Figures 5 to 8 . We assume for $I(t)$ a time evolution as follows:

$$
I(t)=I\left(t_{\mathrm{f}}\right)+\left(I(0)-I\left(t_{\mathrm{f}}\right)\right) \exp \left(-\left(\frac{t}{\tau}\right)^{n}\right) .
$$

$I(0)$ is the value of $I(t)$ at time 0 , i e., before the $T$-jump. 
The $T$-jump is always performed at time 0 . At time $t_{\mathrm{f}}$, the transformation is achieved, and $I(t)$ has a constant value, $I\left(t_{\mathrm{f}}\right) . I(0)$ and $I\left(t_{\mathrm{f}}\right)$ are simply measured on the $I(t)$ curves.

The time $\tau$ is what we already called the characteristic time of the phase transformation. $t_{\mathrm{f}}$ is always greater than $\tau$ : at time $t_{\mathrm{f}}$, the exponential term is equal to 0 . The time $\tau$ and the exponent $n$ are determined by a fitting procedure [18]. The exponential function happens to fit very well all our records: an analysis of the data with more than one characteristic time is not justified. The choice of the exponential function will be justified in a less empirical way in the last section. Anyway, this function appears to be a very suitable tool to quantify the various time evolutions.

3.5.1. Influence of the Initial Temperature. - If the initial temperature $T_{\text {nnt }}$ is too low, i.e., if $T_{\text {trans }}-T_{\text {init }}$ is too large, no phase transition is observed after the $T$-jump. We can infer from Tables II and III that for a given laser energy $E_{1}$, the limit of appearance of a transition is at $T_{\text {trans }}-T_{1 \text { it }}=12^{\circ} \mathrm{C}$. In this way we evaluate the amplitude of the temperature-jump at this energy.

For $10{ }^{\circ} \mathrm{C}<T_{\text {trans }}-T_{\text {mit }}<12{ }^{\circ} \mathrm{C}$, a transition is observed, but the stability of the hightemperature phase is short. Taking as an example the cubic/lamellar transformation corresponding to the curve (a) of Figure 5 (see also Tab. II), we see that $I(t)$ decreases rapidly and that no stable $I\left(t_{\mathrm{f}}\right)$ intensity can be measured. In that case, the power of the IR laser does not provide enough energy to balance the thermal losses during the first seconds after the $T$-jump.

Increasing the initial temperature, i.e., for $T_{\text {trans }}-T_{\text {init }}<10{ }^{\circ} \mathrm{C}$, stable high-temperature phases are induced for several seconds or even longer. The most important fact is that the values of $\tau$, obtained from the fitting procedure, decrease with decreasing $T_{\text {trans }}-T_{\text {init }}$, as can be seen in Table III. $\tau$ varies from about a second to about $100 \mathrm{~ms}$. Corresponding $I(t)$ curves are drawn in Figure 7. For all these data, the values obtained for $n$ are near 1.0, so the different times $\tau$ are comparable.

3.5.2. Influence of the Power of the IR Laser. - For a comparable $T_{\mathrm{int}}$, increasing the power of the IR laser induces faster kinetics. In Figure 6, the two $I(t)$ curves correspond to two $T$-jumps from the cubic phase to the lamellar phase with comparable $T_{\mathrm{nnt}}$, but with two different laser powers. In the case of the higher laser power, the value of $\tau$ is reduced by about a factor 5 , and is in the range of the time-resolution of our experiment. For these very fast transitions we cannot collect a sufficient number of experimental points during the time evolution.

A similar effect in the case of an hexagonal/cubic transformation is shown in Figure 8 . In this case, $\tau$ is reduced by a factor of 3 and our time-resolution is sufficient to determine the kinetics of the transitions.

3.5.3. Value of the Exponent $n$. - The value of $n$ is always between 0.5 and 1.0. Table III shows that $n$ decreases with $T_{\text {trans }}-T_{1 \text { nit }}$, but not at the same rate as $\tau$. For the first $T$-jumps, $n$ is still equal to $1 \pm 0.1$, but $\tau$ varies from $1.25 \mathrm{~s}$ to $0.5 \mathrm{~s}$. For the later $T$-jumps, $\tau$ is small ( $<100 \mathrm{~ms}$ ) and $n$ is slightly higher than 0.5 , between 0.54 and 0.52 . However, $n$ may also take intermediate values such as 0.85 or 0.59 . At present it is difficult to judge whether $n$ varies continuously from 1.0 to 0.5 when decreasing $T_{\text {trans }}-T_{\text {intt }}$, or whether a cross-over of two regimes of growth, one characterised by $n=1.0$ and the other by $n=0.5$ is involved.

In the case of the cubic/lamellar transformations fewer data are available. All the values of $n$ are between 0.78 and 0.52 , with a comparable slight decrease with $T_{\text {trans }}-T_{\text {init }}$. We do not take into account the value $n=1.06$, found in the case of the curve (b) of Figure 6 because at the limit of our time-resolution, the value of $n$ is not measured with a sufficient accuracy. 

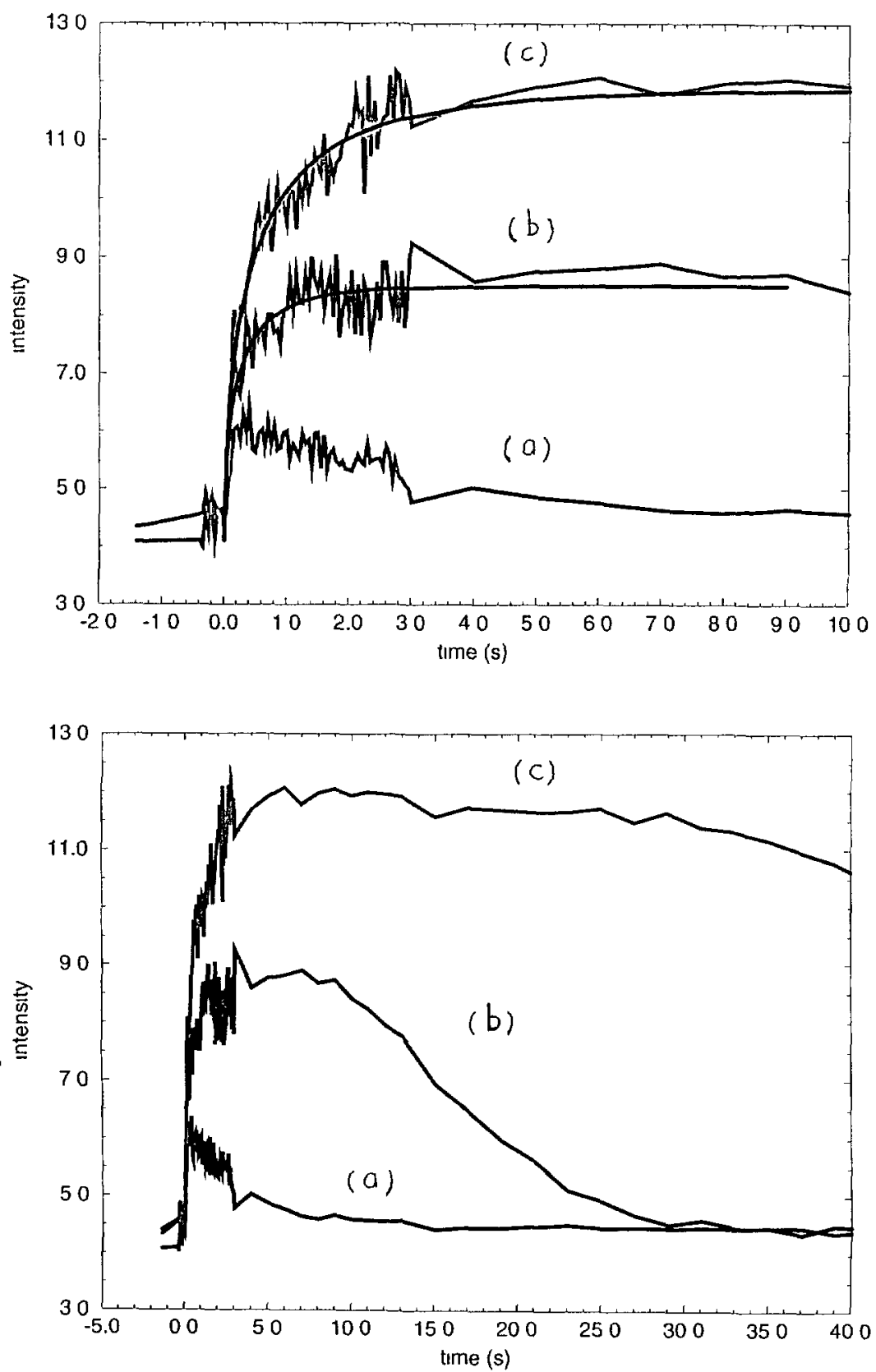

Fig. 5 - Sample A, cubic/lamellar transition: $I(t)$ curves for the same laser power $U_{\text {laser }}=644 \mathrm{~V}$ (about $0.5 \mathrm{~J}$ ) and three different $T_{\text {int }}$ (see Tab II). Two time ranges are shown to underline which time-scales are involved. $I(t)$ increases after the $T$-jump and reaches the value $I\left(t_{\mathrm{f}}\right)$ as the phase transformation is achieved (Section 3). The black curves correspond to the fit using equation in Section 3.5 a) $T_{1 n \jmath t}=33.0^{\circ} \mathrm{C}$. The transition is induced but the lamellar phase is not stable enough and one can't define the intensity $I\left(t_{\mathrm{f}}\right)$. The thermal losses are not compensated for a sufficient duration; b) $T_{\mathrm{tn} 1 \mathrm{t}}=368^{\circ} \mathrm{C}$. A stable lamellar phase is induced, during about $7 \mathrm{~s}\left(t_{\mathrm{f}}=5 \mathrm{~s}\right)$. The fit parameters are $n=0.62$ and $\tau=0.26 \mathrm{~s}$; c) $T_{1 \mathrm{nit}}=38.3^{\circ} \mathrm{C}$ Comparable to b). The lamellar is stable during about $19 \mathrm{~s}\left(t_{\mathrm{f}}=12 \mathrm{~s}\right)$. The fit parameters are: $n=053$ and $\tau=0.57 \mathrm{~s}$. 
Table II. - Cubrc/lamellar $T$-jumps. $T_{1 \mathrm{nit}}$ is the intial temperature of the sample before the $T$-jump. The laser energy is expressed as $U_{\text {laser }}: U_{\text {laser }}=644 \mathrm{~V}$ corresponds to about $0.5 \mathrm{~J}$ and $U_{\text {laser }}=820 \mathrm{~V}$ to about $1.0 \mathrm{~J} . n$ and $\tau$ are the fitted values for the time evolution of $I(t)$ (see Section 3). Sample A: $T_{\text {trans }}=44.64{ }^{\circ} \mathrm{C}$ (see Tab. I). Six T-jumps are shown for the same IR laser power but different $T_{\text {nit }}$. Some of the corresponding $I(t)$ curves are drawn in Fugure 5. Sample $C: T_{\text {trans }}=36.34{ }^{\circ} \mathrm{C}$ (see Tab. I). Two T-jumps are reported with about the same $T_{\text {init }}$ and two different laser powers. The corresponding $I(t)$ curves are drawn in Figure 6.

Sample A

\begin{tabular}{|c|c|c|c|c|}
\hline$T_{\text {init }}$ (C) & $T_{\text {trans }-T_{\text {Init }}(C)}$ & $U_{\text {laser }}(V)$ & $n$ & $\tau(s)$ \\
\hline $\begin{array}{c}335 \\
\text { (a) fig 5 }\end{array}$ & 162 & 644 & - & no transtion \\
\hline 349 & 95 & 644 & - & $\begin{array}{c}\text { transition but no } \\
\text { stability }\end{array}$ \\
\hline $\begin{array}{c}368 \\
\text { (b) fig 5 }\end{array}$ & 78 & 644 & 062 & 047 \\
\hline $\begin{array}{c}383 \\
\text { (c) fig 5 }\end{array}$ & 63 & 644 & 053 & 057 \\
\hline 383 & 63 & 644 & 052 & 041 \\
\hline
\end{tabular}

Sample C

\begin{tabular}{|c|c|c|c|c|}
\hline$T_{\text {Int }}(\mathrm{C})$ & $T_{\text {trans }}-T_{\text {Intt }}(C)$ & Ulaser $(V)$ & $n$ & $\tau(s)$ \\
\hline $\begin{array}{c}335 \\
\text { (a) fig 6 }\end{array}$ & 29 & 650 & 067 & 01 \\
\hline $\begin{array}{c}340 \\
\text { (b) fig 6 }\end{array}$ & 24 & 820 & $(106)$ & 002 \\
\hline
\end{tabular}

All data demonstrate that the rates of the phase transformation strongly depend on the final temperature after the jump, i.e., on the degree of superheating. Faster kinetics is always observed on increasing either $T_{\text {nnt }}$ at a given laser energy or on increasing the laser energy at a constant $T_{\text {init }}$. 


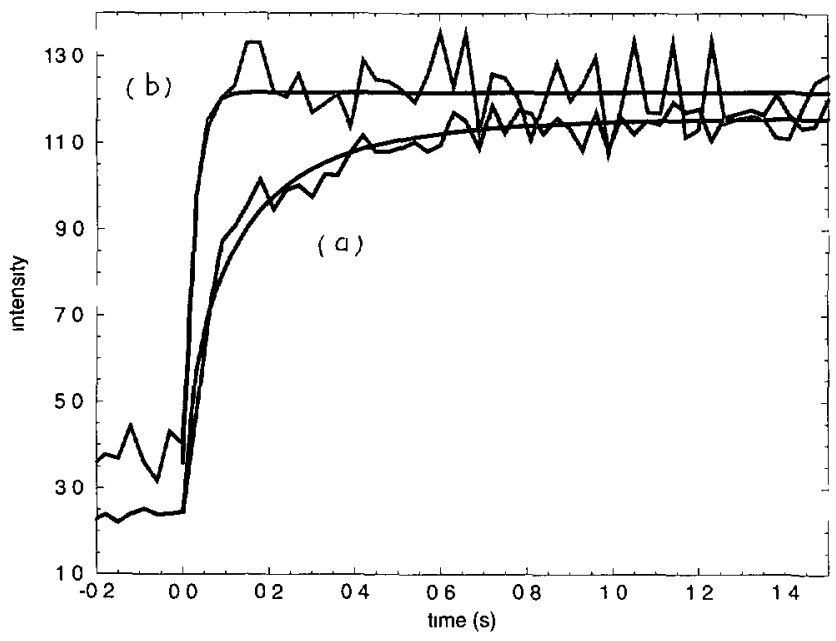

Fig. 6. - Sample C, cubic/lamellar transition: $I(t)$ curves for very similar $T_{\text {int }}$ and two different laser powers (see Tab. II). a) $T_{\text {1nit }}=33.5^{\circ} \mathrm{C}, U_{\text {laser }}=650 \mathrm{~V}$ (about $0.5 \mathrm{~J}$ ), $n=0.67$ and $\tau=0.1 \mathrm{~s}$; b) $T_{\text {inst }}=34.0^{\circ} \mathrm{C}, U_{\text {laser }}=820 \mathrm{~V}$ (about $1.0 \mathrm{~J}$ ), $n=1.06$ and $\tau=0.02 \mathrm{~s}$. The value of $\tau$ is reduced by a factor 5. In the case of $b$ ), $\tau$ is in the time-range of the resolution of the experiment: the fit is made using very few data points and the value of $n$ is not calculated with good accuracy.

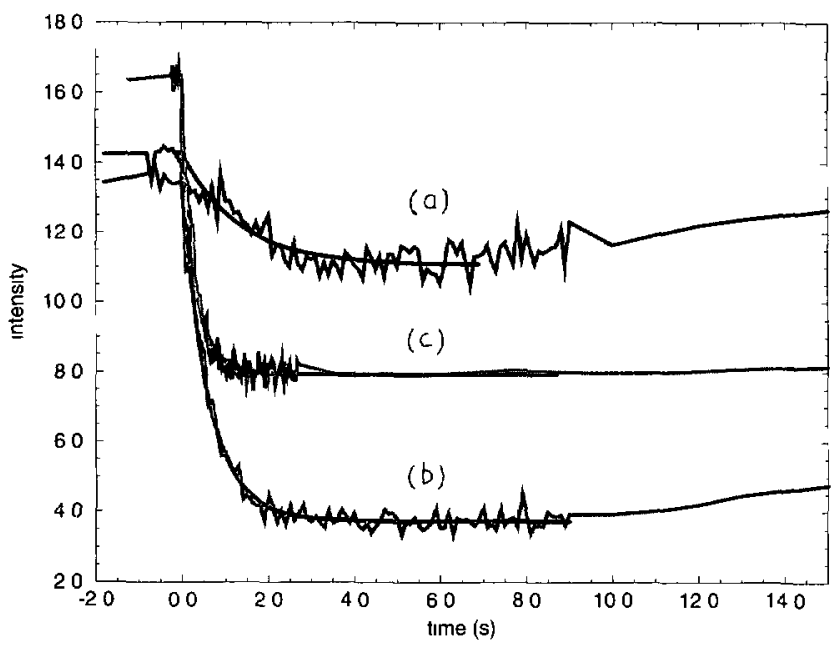

Fig. 7 - Sample C, hexagonal/cubic transition: $I(t)$ curves for the same laser power $U_{\text {laser }}=650$ $\mathrm{V}$ (about $05 \mathrm{~J}$ ) and three different $T_{1 \mathrm{nit}}$ (see Tab. III). The value of $\tau$ decreases as $T_{\mathrm{mit}}$ is increasing. The value of $n$ is decreasing very slowly. a) $T_{\text {nnit }}=13.7^{\circ} \mathrm{C}, U_{\text {laser }}=650 \mathrm{~V}, n=1.05$ and $\tau=1.25 \mathrm{~s}$; b) $T_{\text {int }}=18.9^{\circ} \mathrm{C}, U_{\text {laser }}=650 \mathrm{~V}, n=0.96$ and $\tau=0.58 \mathrm{~s}$; c) $T_{\text {intt }}=20.3^{\circ} \mathrm{C}, U_{\text {laser }}=650 \mathrm{~V}, n=0.85$ and $\tau=0.26 \mathrm{~s}$.

\section{Interpretation of the Results}

During each phase transformation, the coexistence of both phases is clearly observed. The evolution of the diffraction patterns demonstrates that the high-temperature phase is growing 


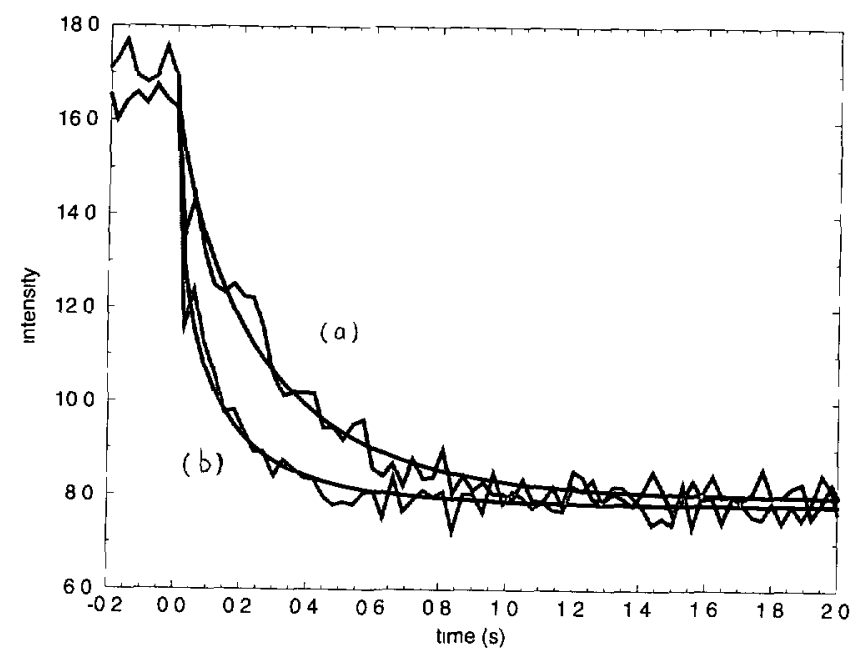

Fig. 8. - Sample C, hexagonal/cubic transition: $I(t)$ curves for very similar $T_{\text {intt }}$ and two different laser powers (see Tab. III). a) $T_{1 \mathrm{nit}}=20.3^{\circ} \mathrm{C}, U_{\text {laser }}=650 \mathrm{~V}$ (about $0.5 \mathrm{~J}$ ), $n=085$ and $\tau=0.26 \mathrm{~s}$; b) $T_{\text {nnit }}=206{ }^{\circ} \mathrm{C}, U_{\text {laser }}=810 \mathrm{~V}$ (about $1.0 \mathrm{~J}$ ), n=0.59 and $\tau=0.075 \mathrm{~s}$. The value of $\tau$ is reduced by a factor 3 .

as the low-temperature phase is disappearing. We will now relate this time evolution to that of the volume fractions of both phases.

We call $\phi(t)$, the volume fraction at a time $t$ after the $T$-jump of the high-temperature phase. As only two phases coexist, the volume fraction of the low-temperature phase is simply $1-\phi(t)$. How does $\phi(t)$ vary versus time?

First of all, we assume that a nucleation/growth mechanism is involved in the phase transformations we are studying. Germs of the high-temperature phase nucleate at some points of the sample then grow to fill completely the volume when the transition is achieved, as depicted in Figure 9. This is consistent with the fact that the transitions are first-order ones and with all our observations.

Avrami has shown [19] that $\phi(t)=1-\exp \left(-\phi_{\text {tot }}(t)\right)$, where $\phi_{\text {tot }}(t)$ is a fictitious volume fraction. The Avrami relation describes quantitatively the change of $\phi(t)$ when different germs touch each other (see Fig. 9) and then stop growing. $\phi_{\text {tot }}(t)$ is a volume fraction calculated by assuming formally that all the germs can "overlap" when still growing as if they were isolated. Notice that this relation is valid without any hypothesis about the nucleation and the growth mechanism.

We make now a second assumption to relate $I(t)$ and $\phi(t)$. We recall that $I(t)$ is the sum of two contributions, the intensities of the first peaks of the low-temperature phase $I_{\mathrm{LT}}(t)$, and of the high-temperature phase $I_{\mathrm{HT}}(t): I(t)=I_{\mathrm{LT}}(t)+I_{\mathrm{HT}}(t)$. We assume that $I_{\mathrm{LT}}(t)$ and $I_{\mathrm{HT}}(t)$ depend linearly on their respective volume fractions, with an additional constant term. We obtain the following simple relations, where Greek letters represent constants:

$$
\left\{\begin{array}{l}
I_{\mathbf{L T}}(t)=\alpha(1-\phi(t))+\beta \\
I_{\mathrm{HT}}(t)=\gamma \phi(t)+\delta
\end{array} \Rightarrow I(t)=\lambda \phi(t)+\mu\right.
$$

Notice that we neglect a possible interference term between the scattering of the two phases: each intensity is proportional only to its own volume fraction. Assuming that $\phi(0)=0$ and 
Table III. - Hexagonal/cubıc T-jumps. Notations are the same as in Table II. Sample C: $T_{\text {trans }}=23.5 \pm 0.5{ }^{\circ} \mathrm{C}$ (see Tab. I). Some of the corresponding $I(t)$ curves are drawn in Frgures 7 and 8.

Sample C

\begin{tabular}{|c|c|c|c|c|}
\hline$T_{\text {Init }}(\mathrm{C})$ & $T_{\text {trans }}-T_{1 \text { Int }}(C)$ & Ulaser $(\mathrm{V})$ & $n$ & $\tau(\mathrm{s})$ \\
\hline 113 & 122 & 650 & - & no transition \\
\hline $\begin{array}{r}137 \\
\text { (a) fig } 7 \\
\end{array}$ & 98 & 650 & 105 & 125 \\
\hline 189 & 46 & 650 & 110 & 085 \\
\hline $\begin{array}{c}189 \\
\text { (b) } \mathrm{fig} 7\end{array}$ & 46 & 650 & 096 & 058 \\
\hline 189 & 46 & 650 & 100 & 049 \\
\hline $\begin{array}{c}203 \\
\text { (c) fig } 7 \text {, (a) fig } 8\end{array}$ & 32 & 650 & 085 & 026 \\
\hline $\begin{array}{l}206 \\
\text { (b) fig } 8 \\
\end{array}$ & 29 & 810 & 059 & 0075 \\
\hline 216 & 19 & 820 & 052 & 009 \\
\hline 221 & 14 & 825 & 054 & 007 \\
\hline
\end{tabular}

that $\left.1-\phi\left(t_{\mathrm{f}}\right)\right) \ll 1$, we obtain: $I(t)=I(0)+\left(I\left(t_{\mathrm{f}}\right)-I(0)\right) \phi(t)$. By comparing with the function we use to fit the $I(t)$ curves in Section 3, we find: $\phi(t)=1-\exp \left(-\left(\frac{t}{\tau}\right)^{n}\right)$. This expression is the Avrami relation, written for a fictitious volume fraction $\phi_{\text {tot }}(t)$ equal to $\left(\frac{t}{\tau}\right)^{n}$. This functional dependence of $\phi_{\text {tot }}(t)$ is the usual one [20].

The interpretation of the values of $\tau$ and $n$ is not straightforward. The major difference is that by varying the initial temperature at a given laser energy $n$ remains constant (close to 1 ) which is not the case if the laser energy is varied at a given $T_{\text {intt }}$. The difficulty arises because the expression of $\phi_{\mathrm{tot}}(t)$ is a mixture of three components: the rate of nucleation, the shape of the germs, and their rate of growth [20]. If all the germs have the same shape and the same rate of growth, $\phi_{\text {tot }}(t)$ can be written as: $\phi_{\text {tot }}(t)=\frac{1}{V} \int_{0}^{t} i\left(t^{\prime}\right) v\left(t-t^{\prime}\right) \mathrm{d} t^{\prime}$, where $\imath\left(t^{\prime}\right) \mathrm{d} t^{\prime}$ is the 

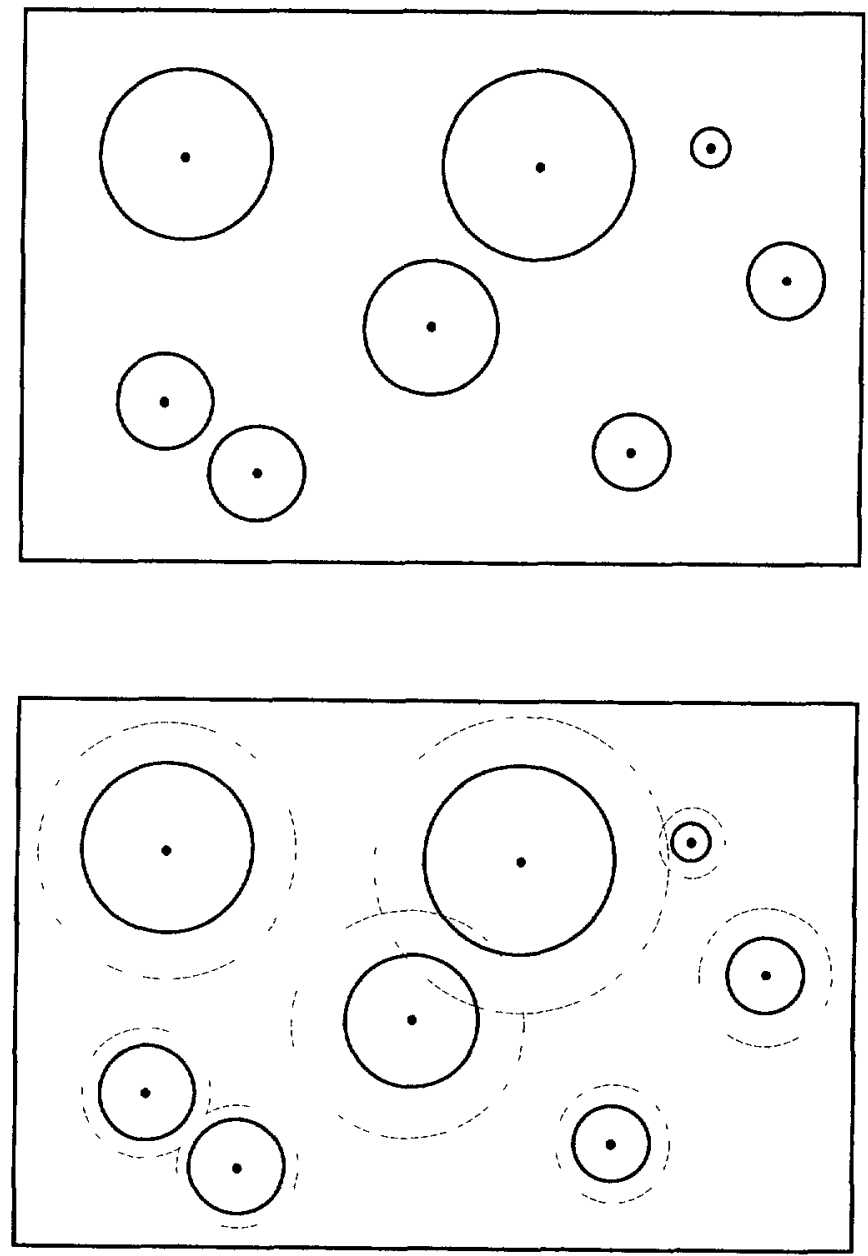

Fig. 9. - Nucleation/growth mechanism. Avrami relation, redrawn from [20]. a) At the beginning of the growth, $\phi(t)$ and $\phi_{\text {tot }}(t)$ are equal. They are symbolised by the sum of the area of the circles; b) If the different germs touch each other, $\phi(t)$ and $\phi_{\text {tot }}(t)$ are different. $\phi(t)$ is the real volume fraction occupied by the germs. $\phi_{\text {tot }}(t)$ is the fictitious area corresponding to the sum of the area of the dashed circles.

number of germs created between the time $t^{\prime}$ and $t^{\prime}+\mathrm{d} t^{\prime}, v\left(t-t^{\prime}\right)$ the volume of a germ at time $t$ and $V$ the total volume. $v(t)$ is the volume of an isolated germ at a time $t$ after the beginning of its growth. To proceed further we have to make another assumption. We consider only the simplest case when all the germs begin to grow at time $t=0$. However, this choice can't be proved from our experiments but we just want to obtain a qualitative interpretation of $\tau$ and $n$.

For a very rapid nucleation process, we take $\imath(t)=N \delta(t) ; N$ is the total number of germs, which remains constant. The expression for $\phi_{\text {tot }}(t)$ reduces to: $\phi_{\text {tot }}(t)=\frac{N}{V} v(t)=g v(t)$, where $g$ is the number of germs per unit volume. Models for $v(t)$ give [20] $v(t)=K t^{n_{0}}$, so: $\phi_{\text {tot }}(t)=g K t^{n_{0}}$. In such a simple case, the exponent $n$ is equal to $n_{0}$, the exponent for an 
isolated germ, and $\tau=\frac{1}{(g K)^{1 / n_{0}}}$.

The observed decrease of $\tau$ can then be interpreted as an increase of either $g$, the number of germs per unit volume, or $K$, which is related to the rate of growth of an isolated germ. It seems reasonable that an increase of the power delivered by the IR laser could increase $g$, or that $K$ depends on the quantity $T_{\text {trans }}-T_{\text {mit }}$. The quite small value for $n$ suggests an anisotropic shape of the germs (plates or cylinders) [20]. The hypothesis of identical germs appearing at the same time $t=0$ implies a well defined value $n_{0}$ for the exponent $n$. However we observe an evolution of $\mathrm{n}$ from 1.0 to 0.5 . This might be explained by differences in the nucleation rates. In other words, the function $\imath(t)$ is not a delta function of time (all germs do not appear exactly at the same time $t=0$ ) and is different from 0 during the whole nucleation process. Then, the integration over time gives for $\phi_{\text {tot }}(t)$ an exponent $n$ different from $n_{0}$.

This interpretation in the framework of a nucleation/growth mechanism is in good agreement with all our observations by time-resolved X-ray diffraction. We can obtain from our results the dependence of $\phi_{\text {tot }}(t)$ in terms of the two parameters $\tau$ and $n$. These last two quantities can be related to the nucleation process (via the function $\imath(t)$, or the number density of germs, $g$ ), to the shape of the germs and to their rate of growth (constant $K$ and exponent $n_{0}$ ). But as already mentioned, we are not able to obtain all these last quantıties separately. Simple additional assumptions can just indicate that the different rates of phase transformation we observe can certainly be understood in this framework.

\section{Conclusion}

The experiments show that the $T$-jump technique together with time-resolved $\mathrm{X}$-ray detection is a powerful technique to study phase transitions. At the time scale of our measurements we observe a nucleation/ growth mechanism for the phase transformations, as expected for first-order transitions. The characteristic time can vary from seconds to less than $100 \mathrm{~ms}$. The relatively small value of the exponent $n$ could indicate that the germs have anisotropic shapes.

Clearly, our results are mainly explained by the first-order type of the transitions. Unfortunately, the microscopic mechanisms involved are too fast to be detected with the timeresolution we have. The fact that the diffraction pattern has already changed on the first record immediately after the $T$-jump shows that the microscopic transformations occur within $50 \mathrm{~ms}$. The study of the very beginning of the nucleation process, and of any possible connected structural change would require investigation of the ms time-scale, at most.

The comparison of the experiments with different $T_{\mathrm{mit}}$ and different powers of the IR laser provides accurate information on the principle of a $T$-jump experiment itself. We analyse quantitatively the rate of the phase transformations by using exponential fits. We propose a relation between the intensity of a diffraction peak and the volume fraction of both phases. The optical observation of the shape of the germs during their growth after a $T$-jump would be a good complementary experiment and should enable us to improve our calculations of Section 4 quantitatively.

\section{Acknowledgments}

We are very grateful to D. Dalle (Laboratorre de Physique des Solides, Orsay) for his ingenious rotation device of the capillaries. 


\section{References}

[1] Clunie J.S., Goodman J F. and Symons P.C., Trans. Faraday Soc. 65 (1969) 287.

[2] Israelachvili J.N., Mitchell J. and Ninham B.W., J. Chem. Soc., Faraday Trans. II 72 (1976) 1523-1568.

[3] Rancon Y. and Charvolin J., J Phys Chem. 92 (1988) 2646.

[4] Clerc M., Levelut A.M. and Sadoc J.F., J Phys II France 1 (1991) 1263-1276.

[5] Sotta P., J. Phys. II France 1 (1991) 763-772.

[6] In addition to the equality of the three spacings $\left(d_{100 \text { hex }}=d_{211 \mathrm{cub}}=d_{100 \text { lam }}\right)$ there is a second epitaxial relation. $a[1,1,1]$ direction of the cubic phase is parallel to the hexagonal director and perpendicular to the lamellar director.

[7] Knight P., Wyn-Jones E. and Tiddy G.J.T., J. Phys. Chem. 89 (1985) 3447-3449.

[8] Laggner P., Kriechbaum M. and Rapp G., J. Appl. Cryst 24 (1991) 836-842.

[9] Rapp G., Rappolt M. and Laggner P., Prog. Coll. Polym. Scı 93 (1993) 25-29.

[10] NIKKO Chemicals Company, 1, 4, 8 Nihonbashi-Badurocko. Chuoko, Tokyo 103, Japan.

[11] Rapp G., Goody R.S., J. Appl. Cryst 24 (1991) 857-865.

[12] Rapp G., Acta Phys Polonıca A 82 (1992) 103-120.

[13] Waggener W.C., Anal. Chem. 30 (9) (1958).

[14] IR Spectroscopy Service, Laboratoire de Chimie Inorganique, Bâtiment 420, Université Paris-Sud, Orsay, France

[15] Gabriel A, Rev. Scr. Instrum 48 (1977) 1303-1305.

[16] Boulin C., Kempf R., Gabriel A. and Koch M.H.J., Nucl. Instrum Methods. A 269 (1988) 312320.

[17] Boulin C., Kempf R, Gabriel A, Koch M.H.J. and McLaughlin S.M., Nucl. Instrum. Methods. A 249 (1986) 399-407.

[18] Turner P, Oregon Graduate Institute (pturner@amb4.ccalmr.ogi.edu)

[19] Avramı M., J. Chem Phys. 7 (1939) 1103; 8 (1940) 212; 9 (1941) 177.

[20] Doremus R.H., Rate of Phase Transformations (Academic Press, 1985). 